Working Papers

Institute of

Mathematical

Economics

November 2005

\title{
On Core Membership Testing for Hedonic Coalition Formation Games
}

Shao Chin Sung and Dinko Dimitrov

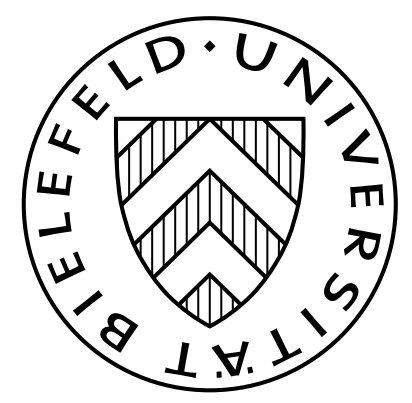

IMW - Bielefeld University

33501 Bielefeld · Germany

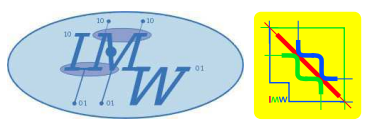

email: imw@wiwi.uni-bielefeld.de

http://www.wiwi.uni-bielefeld.de/ imw/Papers/showpaper.php?374

ISSN: 0931-6558 


\title{
On core membership testing for hedonic coalition formation games
}

\author{
Shao Chin Sung* \\ Department of Industrial and Systems Engineering \\ Aoyama Gakuin University \\ 5-10-1 Fuchinobe, Sagamihara City, Kanagawa, 229-8551, Japan \\ Email: son@ise.aoyama.ac.jp \\ Dinko Dimitrov \\ Institute of Mathematical Economics \\ Bielefeld University \\ P.O. Box 100131, 33501 Bielefeld, Germany \\ Email: d.dimitrov@wiwi.uni-bielefeld.de
}

November 21, 2005

\begin{abstract}
We are concerned with the problem of core membership testing for hedonic coalition formation games, which is to decide whether a certain coalition structure belongs to the core of a given game. We show that this problem is co-NP complete when players'preferences are additive.

JEL Classification: C71, C63.

Keywords: additivity, coalition formation, core, co-NP completeness, hedonic games.
\end{abstract}

${ }^{*}$ Corresponding author. 


\section{Introduction}

The study of computational complexity in hedonic coalition formation games, or simply hedonic games, has a short history, although these issues in cooperative and non-cooperative game theory are being gradually recognized. Maybe the reason is that the formal model of a hedonic game was only recently introduced (cf. Banerjee, Konishi, and Sönmez (2001) and Bogomolnaia and Jackson (2002)). This model consist of a finite set of players and a preference relation for each player defined over the set of all coalitions containing the corresponding player. The outcome of a hedonic game is a coalition structure (i.e., a partition of the set of players into coalitions). A coalition structure is called stable if there is no group of individuals who can all be better off by forming a new deviating coalition. The core of a hedonic game is the collection of all stable coalition structures.

Computational complexity issues related to hedonic games in a general setting are studied by Ballester (2004). As shown by this author, the problem to decide whether a given hedonic game has a nonempty core is NPcomplete $^{1}$. Cechlarová and Hajduková $(2002,2004)$ and Dimitrov, Borm, Hendrickx, and Sung (2004) also elaborate on the computational complexity of core related solution concepts for hedonic games but in a less general setting, i.e., in games with some restrictions imposed on players'preferences. In particular, Dimitrov, Borm, Hendrickx, and Sung (2004) consider preference profiles based on aversion to enemies that consitute a small subdomain of the domain of additive preferences, and show that finding a core member for such games is NP-complete. The corresponding preference domains are formally introduced in the next section.

\footnotetext{
${ }^{1}$ For an introduction to computational complexity, definitions of NP, NP-complete, NP-hard, and a catalog of NP-complete problems, we refer to Garey and Johnson (1979).
} 
In this note we consider the problem of core membership testing for hedonic games. Given a hedonic game and a coalition structure, the problem of core membership testing is to decide whether the coalition structure belongs to the core of the game. We show that this problem is co-NP complete when players' preferences are additive. Indeed, the co-NP completeness is shown by a reduction to hedonic games in which players'preferences are based on aversion to enemies. Hence, the preference domain based on aversion to enemies turns out to have a referential role with respect to this computational complexity issue.

\section{Preliminaries}

Let $N=\{1, \ldots, n\}$ be a finite set of players. A coalition is a nonempty subset of $N$. For each player $i \in N$, we denote by $\mathcal{A}^{i}=\{X \subseteq N \mid i \in X\}$ the collection of all coalitions containing $i$. A collection $\Pi$ of coalitions is called a coalition structure if $\Pi$ is a partition of $N$, i.e., all coalitions in $\Pi$ are pairwise disjoint and $\bigcup_{X \in \Pi} X=N$. We denote by $\mathcal{C}^{N}$ the collection of all coalition structures. For each coalition structure $\Pi \in \mathcal{C}^{N}$ and each player $i \in N$, we denote by $\Pi(i)$ the coalition in $\Pi$ which contains $i$, i.e., $\Pi(i) \in \Pi \cap \mathcal{A}^{i}$.

We assume that each player $i \in N$ is endowed with a preference $\succeq_{i}$ over $\mathcal{A}^{i}$, i.e., a binary relation over $\mathcal{A}^{i}$ which is reflexive, complete, and transitive. Moreover, we assume that the preference of each player $i \in N$ over $\mathcal{C}^{N}$ is purely hedonic, i.e., it is completely characterized by $\succeq_{i}$ in such a way that, for each $\Pi, \Pi^{\prime} \in \mathcal{C}^{N}$, player $i$ weakly prefers $\Pi$ to $\Pi^{\prime}$ if and only if $\Pi(i) \succeq_{i} \Pi^{\prime}(i)$.

A hedonic game is a pair $\langle N, \succeq\rangle$ of a finite set $N$ of players and a profile $\succeq=\left(\succeq_{1}, \ldots, \succeq_{n}\right)$ of players' preferences. We denote by $\mathcal{G}$ the collection of 
all hedonic games. Let $\langle N, \succeq\rangle \in \mathcal{G}$. We say that a coalition $X$ is a deviation from a coalition structure $\Pi$ in $\langle N, \succeq\rangle$ if $X \succ_{i} \Pi(i)$ for each $i \in X$. We say that a coalition structure $\Pi$ is stable in $\langle N, \succeq\rangle$ if no deviation from $\Pi$ exists, i.e., for each coalition $X$, there exists $i \in X$ satisfying $\Pi(i) \succeq_{i} X$. The core of a hedonic game $\langle N, \succeq\rangle$, denoted by $\Phi(N, \succeq)$, is the collection of all coalition structures which are stable in $\langle N, \succeq\rangle$.

Let $\succeq=\left(\succeq_{1}, \ldots, \succeq_{n}\right)$ be a preference profile. We say that $\succeq$ is additive if, for each $i \in N$, there exists a function $v_{i}: N \rightarrow \mathbb{R}$ characterizing $\succeq_{i}$ in such a way that, for all $X, Y \in \mathcal{A}^{i}$,

- $X \succeq_{i} Y$ if and only if $\sum_{j \in X} v_{i}(j) \geq \sum_{j \in Y} v_{i}(j)$

For simplicity, by $v_{i}(X)$ we denote $\sum_{j \in X} v_{i}(j)$ for each $i \in N$ and for each $X \in \mathcal{A}^{i}$.

Given an additive preference profile $\succeq$ and any two players $i, j \in N$, we say that $j$ is a friend (enemy) of $i$ if and only if $v_{i}(j)>0\left(v_{i}(j)<0\right)$; if $v_{i}(j)=0$, and we say that $j$ is a neutral coalitional partner of $i$. Finally, we say that a preference profile $\succeq$ is based on aversion to enemies if $\succeq$ is additive, and for each $i \in N, v_{i}(\cdot) \in\{-n, 1\}$ with $v_{i}(i)=1$. Hence, restricting players' preferences in such a way displays a situation in which each player $i \in N$ has very strong enemies, very weak friends, and no neutral coalitional partners.

\section{Core membership testing}

In this section we study the problem of core membership testing formulated as follows:

\section{The Problem of Core Membership Testing (CMT)}

Given: A hedonic game $\langle N, \succeq\rangle \in \mathcal{G}$ and a coalition structure $\Pi \in \mathcal{C}^{N}$. 
Question: Is $\Pi \in \Phi(N, \succeq)$ ?

This problem belongs to the complexity class co-NP, i.e., the complexity class containing the complements of the decision problems in the complexity class NP. The complement of a decision problem is defined as the problem with the "YES" and "NO" answer reversed. The complement of the CMT problem can then be described as follows. Given a hedonic game $\langle N, \succeq\rangle \in \mathcal{G}$ and a coalition structure $\Pi \in \mathcal{C}^{N}$, and ask whether $\Pi \notin \Phi(N, \succeq)$, i.e., whether there is a deviation $X$ from $\Pi$ in $\langle N, \succeq\rangle$. This problem, the complement of $\mathrm{CMT}$, belongs to NP, because in polynomial time of $n$ one can

(1) guess non-deterministically a coalition $X$,

(2) test deterministically whether $X$ is a deviation from $\Pi$ in $\langle N, \succeq\rangle$, and

(3) the answer is "YES" if some coalition $X$ is a deviation from $\Pi$ in $\langle N, \succeq\rangle$, and otherwise "NO".

Hence, the CMT problem belongs to co-NP.

Before we show that this problem is co-NP complete when players'preference are additive, let us first recall some properties of hedonic games with preference profiles based on aversion to enemies. For more details the reader is referred to Dimitrov, Borm, Hendrickx, and Sung (2004). Let $\langle N, \succeq\rangle \in \mathcal{G}$ be a hedonic game with preference profile $\succeq$ based on aversion to enemies. It is known that

- the core $\Phi(N, \succeq)$ is always nonempty.

In order to describe the properties of core members, let us introduce some terminology. Let $H=(V, E)$ be a (undirected) graph, where $V$ is the set of vertices and $E$ is the set of edges, i.e., each edge is a set consisting of 
two different vertices from $V$. A clique $X$ in $H$ is a subset of $V$ such that $\{i, j\} \in E$ for each $i, j \in X$ with $i \neq j$.

Let $H_{\langle N, \succeq\rangle}=(V, E)$ be a (undirected) graph with

- $V=N$, and

- $E=\left\{\{i, j\} \subseteq V \mid i \neq j\right.$ and $\left.v_{i}(j)=v_{j}(i)=1\right\}$,

and let $\Pi \in \mathcal{C}^{N}$ be a core member, i.e., $\Pi \in \Phi(N, \succeq)$. Then, it is known that

- each $X \in \Pi$ is a clique in $H_{\langle N, \succeq\rangle}$.

Suppose $X$ is not a clique in $H_{\langle N, \succeq\rangle}$ for some $X \in \Pi$. Then, $v_{i}(j)=-n$ for some $i, j \in X$, which implies that $v_{i}(X)<v_{i}(i)$. Hence, $X \prec_{i}\{i\}$, and thus, $\{i\}$ is a deviation from $\Pi$ in $\langle N, \succeq\rangle$. Therefore $\Pi \notin \Phi(N, \succeq)$.

Moreover, it is known that

- at least one of the largest cliques in $H_{\langle N, \succeq\rangle}$ belongs to $\Pi$.

Suppose $\Pi$ does not contain any of the largest cliques in $H_{\langle N, \succeq\rangle}$, and let $X$ be one of the largest cliques in $H_{\langle N, \succeq\rangle}$. Then, for each $i \in X$, we have

$$
v_{i}(X)=|X|>|\Pi(i)| \geq v_{i}(\Pi(i)) .
$$

Hence, $X$ is a deviation from $\Pi$ in $\langle N$, $\rangle$, i.e., $\Pi \notin \Phi(N, \succeq)$. It follows from this property that the problem of finding a core member of a given hedonic game, with preference profile based on aversion to enemies, is at least as hard as the problem for finding a largest clique in a given graph, which is a NP-hard optimization problem.

We are now ready to present our result. 
Theorem 1 The problem of core membership testing for hedonic games with additive preference profile is co-NP complete.

Proof. As already mentioned, the CMT problem belongs to co-NP. It suffi ces to show that this problem is co-NP hard. The co-NP hardness is shown by a polynomial time reduction from a co-NP complete problem called the $\overline{\text { CLIQUE }}$ problem, which is the complement of the CLIQUE problem. The CLIQUE problem is defined as follows:

\section{Clique Problem (CLIQUe)}

Given: A graph $G=(V, E)$ and a positive integer $2 \leq K \leq|V|$.

Question: Does $G$ contain a clique of size $K$ ?

Let $(G, K)$ be an instance of the $\overline{\text { CLIQUE }}$ problem, i.e., $G=(V, E)$ is a graph and $K$ is a positive integer such that $2 \leq K \leq|V|$. Define a hedonic game $\langle N, \succeq\rangle$ as follows. Take $N=\{1,2, \ldots, K-1\} \times V$ to be the set of players, and let $n=|N|=(K-1)|V|$. For each $(k, s) \in N$, the preference $\succeq_{(k, s)}$ of player $(k, s)$ is characterized by the function $v_{(k, s)}$, which is defined as follows: For each $(\ell, t) \in N$,

$$
v_{(k, s)}(\ell, t)= \begin{cases}1 & \text { if } s=t \\ 1 & \text { if } k=\ell \text { and }\{s, t\} \in E \\ -n & \text { otherwise }\end{cases}
$$

Observe that the transformation from $(G, K)$ to the game $\langle N, \succeq\rangle$ can be done in $O\left(|V|^{4}\right)$ time. Hence, it is a polynomial time reduction. Moreover, observe that players'preferences are, in fact, based on aversion to enemies.

Next, define $\Pi=\left\{X_{s} \mid s \in V\right\}$ with $X_{s}=\{1,2, \ldots, K-1\} \times\{s\}$ for each $s \in V$. Obviously $\Pi$ is a partition of $N$, i.e., $\Pi$ is a coalition structure. Also notice that each $X_{s}$ is a clique of size $K-1$ in $H_{\langle N, \succeq\rangle}$, because $v_{(k, s)}(\ell, s)=1$ 
for all $k, \ell \in\{1,2, \ldots, K-1\}$ and for each $s \in V$. In the following we show that $\Pi \notin \Phi(N, \succeq)$ if and only if $G$ contains a clique of size $K$.

(1) Suppose that $G$ contains a clique $Y$ of size $K$. Then, for each $k \in$ $\{1,2, \ldots, K-1\},\{k\} \times Y$ is also a clique of size $K$ in $H_{\langle N, \succeq\rangle}$. Hence, each $\{k\} \times Y$ is a deviation from $\Pi$, and therefore $\Pi \notin \Phi(N, \succeq)$.

(2) Suppose $\Pi \notin \Phi(N, \succeq)$. Then there exists a deviation $Z$ from $\Pi$. Since each $X_{s} \in \Pi$ is a clique of size $K-1$ in $H_{\langle N, \succeq\rangle}, Z$ must also be a clique of size at least $K$ in $H_{\langle N, \succeq\rangle}$ in order to be a deviation from $\Pi$. Let $Z^{\prime}$ be a subset of $Z$ of size $K$. Since $Z$ is a clique in $H_{\langle N, \succeq\rangle}, Z^{\prime}$ is also a clique in $H_{\langle N, \succeq\rangle}$. Then, by definition, we have either $k=k^{\prime}$ or $s=s^{\prime}$ for all $(k, s),\left(k^{\prime}, s^{\prime}\right) \in Z^{\prime}$ with $(k, s) \neq\left(k^{\prime}, s^{\prime}\right)$.

We show that $k=k^{\prime}$ and $s \neq s^{\prime}$ for all $(k, s),\left(k^{\prime}, s^{\prime}\right) \in Z^{\prime}$ with $(k, s) \neq$ $\left(k^{\prime}, s^{\prime}\right)$, i.e., $Z^{\prime} \subseteq\{k\} \times V$ for some $k \in\{1,2, \ldots, K-1\}$. Let $(k, s) \in Z^{\prime}$. Then, there exists $\left(k^{\prime}, s^{\prime}\right) \in Z^{\prime}$ such that $s^{\prime} \neq s$, because $\left|Z^{\prime}\right|=K>K-1=$ $\left|X_{s}\right|$, and thus, we have $k^{\prime}=k$. We are done when $K=2$. When $K \geq 3$, there exists $\left(k^{\prime \prime}, s^{\prime \prime}\right) \in Z^{\prime}$ such that $\left(k^{\prime \prime}, s^{\prime \prime}\right) \neq(k, s)$ and $\left(k^{\prime \prime}, s^{\prime \prime}\right) \neq\left(k^{\prime}, s^{\prime}\right)$. When $s^{\prime \prime}=s$, we have $k^{\prime \prime} \neq k$, and thus, we have $k^{\prime \prime} \neq k=k^{\prime}$ and $s^{\prime \prime}=s \neq s^{\prime}$, so that there is no edge between $\left(k^{\prime}, s^{\prime}\right)$ and $\left(k^{\prime \prime}, s^{\prime \prime}\right)$ in $H_{\langle N, \succeq\rangle}$, and $Z^{\prime}$ cannot be a clique in $H_{\langle N, \succeq\rangle}$. The same argument hold when $s^{\prime \prime}=s^{\prime}$. Hence, $k=k^{\prime}$ and $s \neq s^{\prime}$ for all $(k, s),\left(k^{\prime}, s^{\prime}\right) \in Z^{\prime}$ with $(k, s) \neq\left(k^{\prime}, s^{\prime}\right)$. i.e., $Z^{\prime} \subseteq\{k\} \times V$ for some $k \in\{1,2, \ldots, K-1\}$.

Finally, since $Z^{\prime}$ is a clique of size $K$ in $H_{\langle N, \succeq\rangle}$ and $Z^{\prime} \subseteq\{k\} \times V$ for some $k \in\{1,2, \ldots, K-1\}$, we have $\{s, t\} \in E$ for each $(k, s),(k, t) \in Z^{\prime}$. Therefore, $\left\{s \in V \mid(k, s) \in Z^{\prime}\right\}$ is a clique of size $K$ in $G$. 


\section{References}

[1] Ballester, C. (2004): NP-completeness in hedonic games, Games and Economic Behavior 49, 1-30.

[2] Banerjee, S., H. Konishi, and T. Sönmez (2001): Core in a simple coalition formation game, Social Choice and Welfare 18, 135-153.

[3] Bogomolnaia, A. and M. O. Jackson (2002): The stability of hedonic coalition structures, Games and Economic Behavior 38, 201-230.

[4] Cechlarová, K. and J. Hajduková (2004): Stable partitions with $\mathcal{W}$ preferences, Discrete Applied Mathematics 138, 333-347.

[5] Cechlarová, K. and J. Hajduková (2002): Computational complexity of stable partitions with $\mathcal{B}$-preferences, International Journal of Game Theory $31,353-364$.

[6] Dimitrov, D., P. Borm, R. Hendrickx, and S.-C. Sung (2004): Simple priorities and core stability in hedonic games, Social Choice and Welfare, forthcoming.

[7] Garey, M. and D. Johnson (1979): Computers and Intractability. A Guide to the Theory of NP-Completeness, Freeman, New York. 